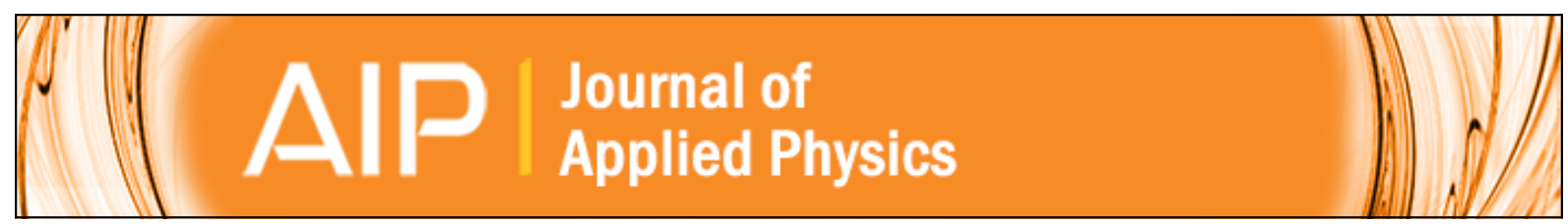

\title{
Granular metamaterials for vibration mitigation
}

G. Gantzounis, M. Serra-Garcia, K. Homma, J. M. Mendoza, and C. Daraio

Citation: Journal of Applied Physics 114, 093514 (2013); doi: 10.1063/1.4820521

View online: http://dx.doi.org/10.1063/1.4820521

View Table of Contents: http://scitation.aip.org/content/aip/journal/jap/114/9?ver=pdfcov

Published by the AIP Publishing

Advertisement:

$\underset{\substack{\text { Aubloning } \\ \text { Alp }}}{A}$ Re-register for Table of Content Alerts

Create a profile.

Sign up today! 


\title{
Granular metamaterials for vibration mitigation
}

\author{
G. Gantzounis, ${ }^{1, a)}$ M. Serra-Garcia, ${ }^{1, b)}$ K. Homma, ${ }^{2, c)}$ J. M. Mendoza, ${ }^{2, d)}$ and C. Daraio ${ }^{3, e)}$ \\ ${ }^{1}$ Graduate Aerospace Laboratories (GALCIT), California Institute of Technology, Pasadena, \\ California 91125, USA \\ ${ }^{2}$ United Technologies Research Center, 411 Silver Lane, East Hartford, Connecticut 06108, USA \\ ${ }^{3}$ Department of Mechanical and Process Engineering (D-MAVT), ETH Zürich, Switzerland and Graduate \\ Aerospace Laboratories (GALCIT), California Institute of Technology, Pasadena, California 91125, USA
}

(Received 23 June 2013; accepted 21 August 2013; published online 6 September 2013)

\begin{abstract}
Acoustic metamaterials that allow low-frequency band gaps are interesting for many practical engineering applications, where vibration control and sound insulation are necessary. In most prior studies, the mechanical response of these structures has been described using linear continuum approximations. In this work, we experimentally and theoretically address the formation of low-frequency band gaps in locally resonant granular crystals, where the dynamics of the system is governed by discrete equations. We investigate the quasi-linear behavior of such structures. The analysis shows that a stopband can be introduced at about one octave lower frequency than in materials without local resonances. Broadband and multi-frequency stopband characteristics can also be achieved by strategically tailoring the non-uniform local resonance parameters. @ 2013 AIP Publishing LLC. [http://dx.doi.org/10.1063/1.4820521]
\end{abstract}

\section{INTRODUCTION}

Metamaterials, defined as artificial structures that exhibit physical properties not available in natural materials, ${ }^{1,2}$ gain their unique properties primarily from their subwavelength spatial arrangement, rather than their composition. Metamaterials had initially been developed for electromagnetic applications such as negative refractive index ${ }^{3}$ and lowfrequency band gaps. ${ }^{4-7}$ The pioneering work of Liu et $a l^{8}$ enabled the development of a novel class of metamaterials, termed locally resonant sonic materials that support lowfrequency band gaps in elastic waves traveling in such media. Many interesting applications have recently been proposed for metamaterials in the field of elastic wave propagation. These include negative effective density, which has been theoretically and experimentally validated, ${ }^{9-13}$ negative effective stiffness, ${ }^{13-15}$ negative refractive index ${ }^{16-20}$ structures, and mechanical energy trapping materials. ${ }^{22}$

A unique feature of locally resonant metamaterials is the ability to form low-frequency band gaps, a feature which is promising in the applied field of mechanical vibration mitigation. ${ }^{23-29}$ Vibration mitigation structures have been realized both in one dimension (1D) ${ }^{24-26}$ and two dimensions (2D). ${ }^{23,24}$ In most of these works, the structures are modeled as simple mass/spring systems, and theoretical models are developed that can efficiently handle the behavior of these structures.

Vibration mitigation in common engineering applications is often achieved by using dynamic vibration absorbers (DVA). This idea was introduced as a single-degree-of-freedom (1DoF) system, where a mass was added to the initial mass-spring

\footnotetext{
a)ggantzou@caltech.edu

b) mserraga@caltech.edu

c)hommak@utrc.utc.com

d)MendozJM@utrc.utc.com

e)daraio@ethz.ch
}

system to achieve suppression of vibration at specific frequencies. $^{30}$ DVAs are widely used for vibration mitigation, ${ }^{31,32}$ using not only $1 \mathrm{DoF}^{30,31}$ but also two-degrees-of-freedom $(2 \mathrm{DoF})^{32}$ and generally multiple-degrees-of-freedom $(\mathrm{MDoF})^{33}$ systems. Combining the knowledge established for MDoF systems in connection to metamaterial design has been proven quite fruitful.

In this paper, we further investigate mechanical vibration mitigation in order to bridge the gap between theoretical results and practical engineering limitations. By performing an analysis on the effect of local resonances on the effective mass of the medium, ${ }^{21}$ we explore the connection of metamaterials with local resonators to the on-site spring potential, a linearized form of the sine-Gordon equation, ${ }^{34}$ which makes possible the development of band gaps from almost the static limit. ${ }^{34} \mathrm{We}$ also investigate the connection between the low-frequency band gaps and the antiresonances of a 2DoF system. In addition, we discuss the effect of using viscoelastic materials in such structures, which allow for further dissipation of the waves due to viscous damping. ${ }^{35}$ The interplay of the variable stiffness linear local resonators on a single structure is, also, presented. Finally, we validate the theoretical arguments with an experimental prototype that measures in very good agreement with the numerical predictions.

\section{THEORETICAL INVESTIGATION OF LOCAL RESONATORS}

Let us first consider a simple 2DoF system, namely a mass $m$ connected to a rigid base and to an attached local resonator of mass $M$ through a spring of stiffness $K$, as shown in Fig. 1(a). The system is driven by a prescribed displacement, $u_{d}$, which corresponds to a system driven by a high impedance input, i.e., driven by an effective mass much higher than those of the chain. By writing the equations of motion for the system in the frequency domain, we find that the 
Fourier component of the displacement, $u$, at a given frequency $\omega$, of the mass $m$ is related to the one of the local resonator, $U$, and to that of the prescribed displacement, $u_{d}$, as follows:

$$
\begin{aligned}
-m \omega^{2} u & =k\left(u_{d}-2 u\right)+K(U-u), \\
-M \omega^{2} U & =K(u-U) .
\end{aligned}
$$

The displacement of the local resonator can be expressed through the displacement of the mass $m$, with the help of (1) and the eigenfrequency $\Omega=\sqrt{K / M}$ as

$$
U=\frac{u}{1-(\omega / \Omega)^{2}} .
$$

At low frequencies, the local resonator does not have any effect on the system, since $U-u \approx 0$. Around the local resonance frequency, $\omega \approx \Omega, U / u \rightarrow \infty$, thus, for finite values of $U, u \approx 0$, which is the anti-resonance condition. For high frequencies, i.e., $\omega \gg \Omega, U \approx 0$, and Eq. (1) turns into a linearized form of the sine-Gordon equation. ${ }^{34}$ In other words, the local resonator acts as an on-site spring potential. From the first of Eqs. (1) together with Eq. (2), we obtain the following equation of motion for the mass $m$ :

$$
-m\left(1+\frac{M / m}{1-(\omega / \Omega)^{2}}\right) \omega^{2} u=k\left(u_{d}-2 u\right) .
$$

In this expression, the local resonance appears as a term adding to the effective mass of the bead. At low frequencies, the bead has a mass of $m+M$, since the bead and the resonator move at the same velocity and they act as a single mass. However, in absence of damping, as the driving frequency approaches the natural frequency of the local resonator, the effective mass of the bead increases without bounds, rendering the mass $m$ unable to move, regardless of the applied force, which corresponds to an antiresonance condition. In an experimental system, the effective mass in the antiresonance condition will be bounded by damping, an effect that will be studied in Secs. III-V. Above the eigenfrequency of the local resonator and up to $\Omega \sqrt{1+(M / m)}$, the effective mass of the bead becomes negative, i.e., it accelerates in the opposite direction of the force acting on the bead. For

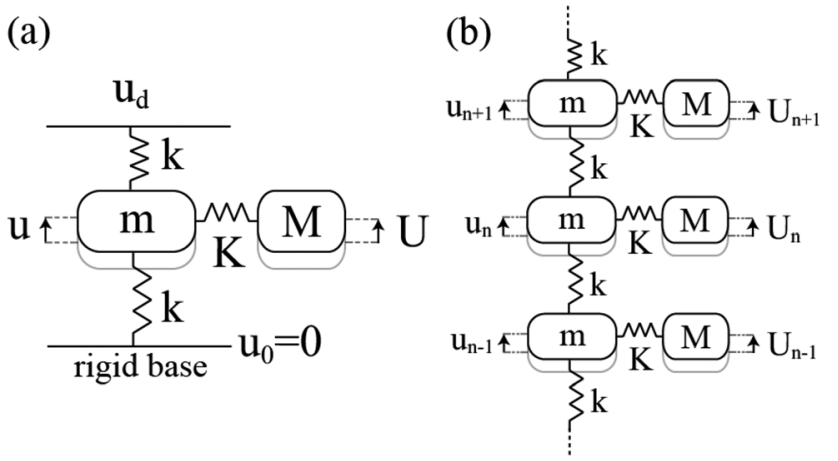

FIG. 1. (a) A mass-spring system connecting two rigid surfaces, consisting of a mass $m$ with an attached local resonator, of mass $M$. (b) A 1D periodic arrangement of the masses and the attached local resonators. frequencies $\omega \gg \Omega$, the second term adding to the effective mass becomes very small, and the effective mass tends to the mass of the bead $m$. However, a more careful investigation reveals that this term should be interpreted as an on-site spring potential, since the frequency variables $\omega$ in the numerator and denominator cancel out, and it becomes a constant stiffness term which can be moved to the right hand side. The displacement of the mass $m$ in terms of the prescribed displacement of the driver can be calculated through Eq. (3). It follows that:

$$
u=u_{d}\left(2-\frac{m \omega^{2}}{k} \frac{\Omega^{2}(1+M / m)-\omega^{2}}{\Omega^{2}-\omega^{2}}\right)^{-1} .
$$

The transmissibility, $T=\left|u(\omega) / u_{0}\right|^{2}$, in decibels is shown in Fig. 2. The anti-resonance condition, $T \rightarrow 0$, is fulfilled at $\omega=\Omega$. There are also two resonances around the antiresonance, as expected, at $0.81 \Omega$ and $3.52 \Omega$. The second resonance is shifted from $(2 \mathrm{k} / \mathrm{m})^{1 / 2}=2.83 \Omega$ to about $[(2 k+K) / m]^{1 / 2}=3.46 \Omega$ due to the local resonator acting as an on-site spring potential that adds to the coupling stiffness.

We now consider an infinite array of point masses $m$, connected through springs of stiffness $k$, to which we attach local resonators of mass $M$ through springs of stiffness $K$, as shown in Fig. 1(b). The displacement of the $n$-th mass, $u_{n}$, is connected to those of the adjacent masses $(n-1$ and $n+1)$ and the $n$-th attached resonator through the equations

$$
\begin{aligned}
-m \omega^{2} u_{n}= & k\left(u_{n+1}+u_{n-1}-2 u_{n}\right)+K\left(U_{n}-u_{n}\right), \\
& -M \omega^{2} U_{n}=K\left(u_{n}-U_{n}\right),
\end{aligned}
$$

with the help of Eq. (6), Eq. (5) takes the form

$$
-m\left(1+\frac{M / m}{1-(\omega / \Omega)^{2}}\right) \omega^{2} u_{n}=-k\left(u_{n+1}+u_{n-1}-2 u_{n}\right) .
$$

We can see that Eq. (7) takes the form of Eq. (3) by assigning $u_{d}=u_{n+1}+u_{n-1}$, i.e., when we consider the driving force as the force experienced by the $n$-th mass due to the neighboring masses. Consequently, the local resonator will strongly attenuate the displacement of the $n$-th mass for $\omega \approx \Omega$, while for $\omega \gg \Omega$, the local resonators act as an on-site spring potential, in the same way as it is already discussed in the case of the $2 \mathrm{DoF}$ system. In order to analyze the propagation of waves in such a structure, we consider solutions of the form $u_{n \pm 1}=u_{n} \exp ( \pm i a)$, with a phase

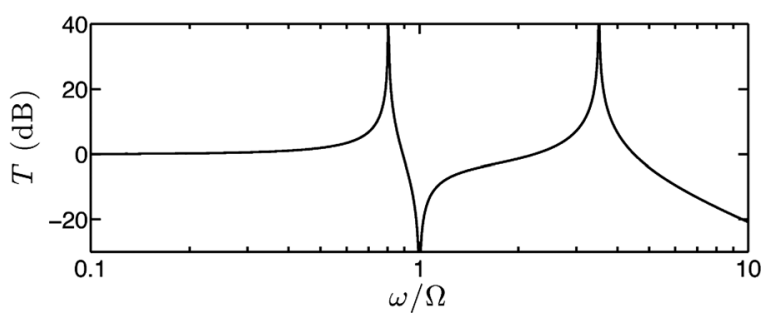

FIG. 2. Transmissibility for the 2DoF system of Fig. 1(a), with $K=k$ and $M=4 m$. 
$-\pi<a<\pi$. The dispersion relation can be derived from Eq. (7) and it takes the form

$$
\sin ^{2}\left(\frac{a}{2}\right)=\left(\frac{\omega}{2 \omega_{0}}\right)^{2} \frac{\Omega^{2}(1+M / m)-\omega^{2}}{\Omega^{2}-\omega^{2}},
$$

where $\omega_{0}=\sqrt{K / M}$ is the natural frequency of the local resonator if the bead to which it is attached is fixed.

The dispersion relation for $K=k$ and $M=4 m$ is shown in Fig. 3(a) with the solid line. A band gap is formed in this case from $0.89 \Omega$ up to $2.24 \Omega$. This band gap can extend to the static limit when $M=\infty$ [thick dashed line of Fig. 3(a)], i.e., for an ideal on site potential, and is also infinitely deep, amounting to the absence of damping. However, in the absence of any local potential $(K=0)$ the usual dispersion relation, with a cutoff at $4 \Omega$ and no low-frequency band gap is observed. It can also be seen that the material with local resonators can be approximated as an on-site spring potential for $\omega>2 \Omega$.

The transmissibility from a finite chain can be calculated using Eq. (5) for $n=1, \ldots, N$, with $u_{0} \equiv 0$ and $u_{N+1} \equiv u_{d}$, which can be written in the matrix form

$$
-\omega^{2} \mathbf{M u}=\mathbf{K u}+\mathbf{F},
$$

with $\mathbf{M}$ the diagonal mass matrix of the system, $\mathbf{K}$ the stiffness matrix of the system, and $\mathbf{F}$ the vector for the external force, with only one nonzero element $F_{N}=k u_{d}$ and $\mathbf{u}=\left[u_{1}, u_{2}, \ldots, u_{N}\right]^{T}$. The transmissibility is then defined as $T=\left|u_{1}(\omega) / u_{1}(\omega=0)\right|^{2}$, for arbitrary value of the driver displacement. The transmissibility for a finite structure of ten masses in the linear chain is shown in Fig. 3(c). The transmissibility decreases exponentially in frequency in the corresponding stop bands in all cases. This exponential decay is proportional to the imaginary part of the phase $a$ in the corresponding band gaps [Fig. 3(b)]. The shape of the attenuation in the local resonance band gap is asymmetric, with the deepest attenuation occurring at the local resonance frequency, $\Omega$, but being still significant across a broad range of frequencies above $\Omega$. It should also be noted that the highfrequency tail of the local resonance attenuation resembles that of the ideal on-site potential (i.e., ground spring).

Figure 4 shows the contour plots of the attenuation coefficient as a function of the mass ratio $M / m$ (Fig. 4(a)) and the
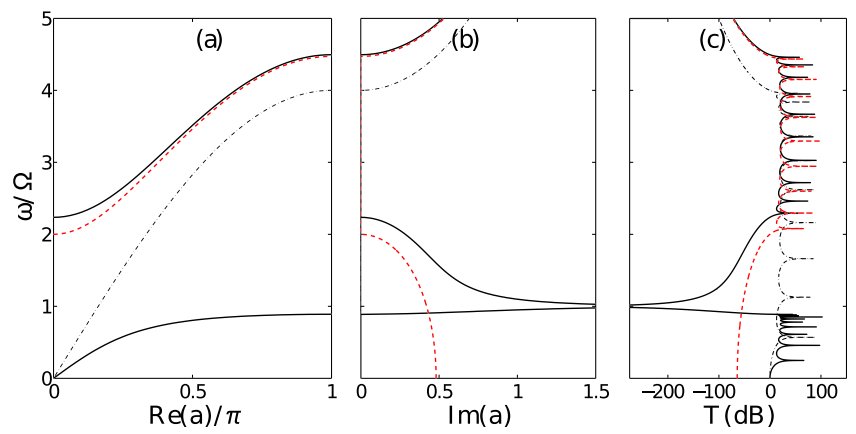

FIG. 3. Real (a) and imaginary (b) parts of the complex band structure of the infinite periodic chain of Fig. 1(b). (c) Transmissibility of a finite chain of ten beads with local resonators (solid black), with no local resonators (dashed red), and with an on-site spring potential (dotted gray).
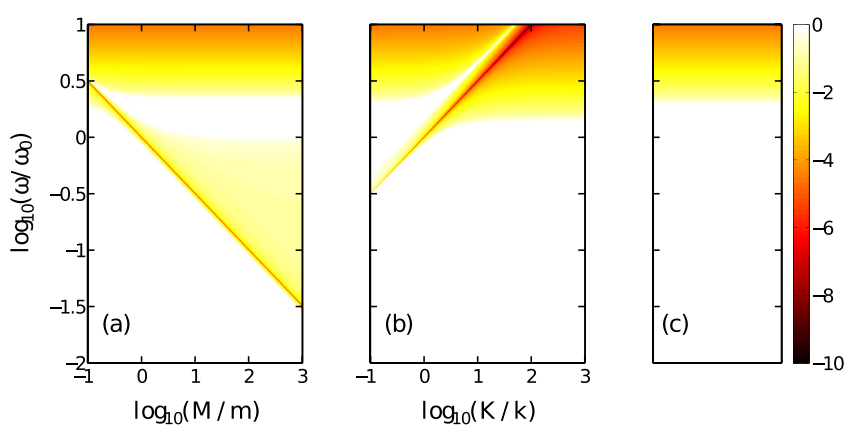

FIG. 4. Color maps of $-\operatorname{Im}(a)$ as a function of the ratio $K / k$ (a), with $M=m$, and of the ratio $M / m$ (b), with $K=k$. The results for a metamaterial without the local resonators is shown in (c) for comparison.

stiffness ratio $K / k$ (Fig. 4(b)), as well as the case with no local resonators (Fig. 4(c)). As $M$ or $K$ changes, the location of the maximum attenuation moves with the local resonance frequency $\Omega$. Figure 4(a) shows that the width of the low frequency band gap extends into lower frequencies as the mass increases. A way of reducing the frequency at which the band gap occurs without increasing the total mass is by making the resonator stiffness, $K$, smaller, as shown in Fig. 4(b). However, this is accompanied by a decrease in the level of attenuation as a side effect.

\section{EFFICACY OF LOCAL RESONANCE BASED METAMATERIAL UNDER MASS AND STIFFNESS CONSTRAINTS}

The vibration attenuation efficacy of the local resonators can be improved by increasing the resonator masses. However, there is often a practical limit to the amount of mass that can be added to the structure. The overall stiffness of the structure also needs to be kept above a certain level to ensure it can support the static load required in practical applications. We investigated vibration blocking performance of the metamaterial with local resonances under mass and stiffness constraints. ${ }^{32}$ In the analyses that follow, the total mass of the structure was constrained to $1.46 \mathrm{~kg}$ and the overall static stiffness to $1.8 \mathrm{~N} / \mu \mathrm{m}$, which are consistent with the actual values for the experimental setup of Fig. 1(b).

In Fig. 5, we consider the effect of damping for a chain of ten beads, as shown in Fig. 1(b). In the case, where the model does not include damping, we can see the strong dip at $1.92 \mathrm{kHz}$, but also the sharp response peaks due to the resonances of the ten beads system. If we include in the model, a loss factor by connecting the beads using a complex stiffness $k_{L}=k(1+i \eta)$ with $\eta=0.2$, we see that most of these resonance peaks are wiped out. However, the sharp dip introduced by the local resonators remains unchanged. If we apply the same damping force to the springs connecting the beads with the local resonators $K_{L}=K(1+i \eta)$, while leaving the contacts undamped, we see that the resonance peaks are also wiped out, but at the price of a shallower bandgap. If the loss factor is applied to both the springs connecting the beads with each other and the springs in the local resonators, the results are similar to the case where the damping is applied to the resonators alone, namely, a shallow band gap 


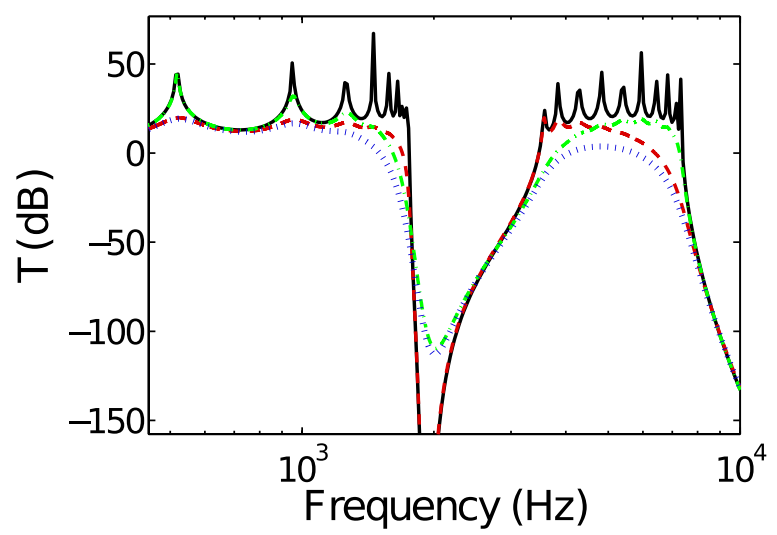

FIG. 5. Transmissibility of the finite structure consisting of ten beads $(m=45 \mathrm{~g}, k=20 \mathrm{~N} / \mu \mathrm{m})$ with local resonators $(M=101 \mathrm{~g}, \Omega / 2 \pi=1.92 \mathrm{kHz})$ shown in Fig. 1(d). The curves depict the four cases with no damping (black solid line), damping only in the main chain (damping factor 0.2 , red dashed line), damping only at the local resonators (damping factor 0.2 , green dasheddotted line), and damping at both the main chain and the local resonators (damping factors 0.2 , blue dotted line).

and no resonance peaks. This last case will be considered for the rest of Sec. III.

We next consider the effect of changing the number of beads along the chain. In Fig. 6(a), we momentarily neglect the aforementioned overall mass and stiffness constraints and consider constant mass and stiffness for each bead, which are $m=0.45 \mathrm{~kg}$ and $k=20 \mathrm{~N} / \mu \mathrm{m}$. The mass of each local resonator is set to the constant value $M=101 \mathrm{~g}$. Without the overall mass and stiffness constraints, we can see that the stop band becomes progressively larger as we increase the number of beads, as expected, since, the heavier the structure, the more it attenuates the input vibration. In Fig. 6(b), we apply the constraint on the total mass of the structure, $1.46 \mathrm{~kg}$, while changing the number of beads. This means that the larger the number of beads, the lighter each bead becomes. In this case, we can see that the stop band deepens when increasing the number of beads, and the cutoff

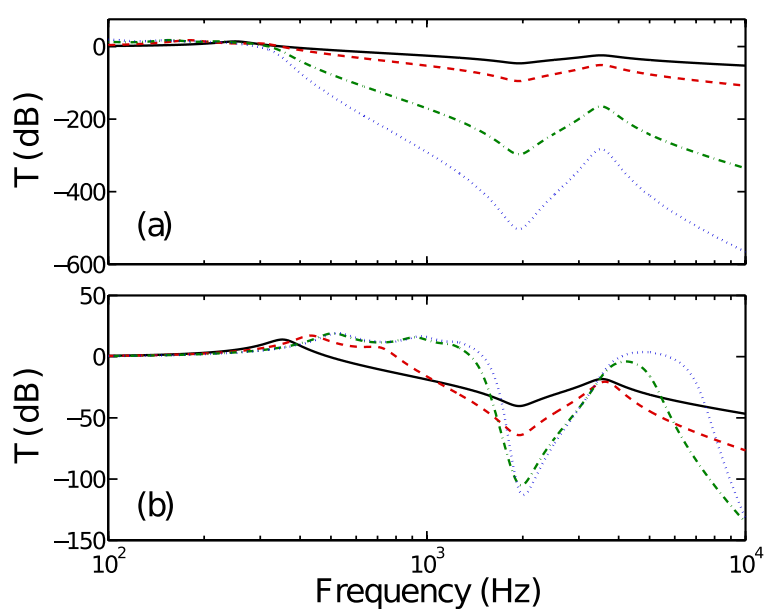

FIG. 6. Transmissibility of a finite structure consisting of different number of beads, $N=1$ (black solid line), 2 (red dashed line), 6 (green dashed dotted line), and 10 (blue dotted line). (a) The mass of each bead and the stiffness of each contact remain constant. (b) The static stiffness and the mass of the whole structure remain constant. The stiffness of the local resonators corresponds to a resonance at $1.92 \mathrm{kHz}$. frequency of the stopband becomes higher in frequency, from $540 \mathrm{~Hz}$ to $1540 \mathrm{~Hz}$, and has a sharper roll-off.

Figure 7(a) shows the effect of changing the mass ratio $M / m$ under constant stiffness for all the springs. These results are complementary to those of Fig. 4 . We can see that, as the mass ratio $M / m$ increases, the width of the stop band widens into high frequencies. Note that the limit $M / m \rightarrow 0$ represents the structure without the attached local resonators. The introduction of the local resonators with the overall mass constraint results in a stop band with a cutoff frequency of about $1.5 \mathrm{kHz}$, which is about one octave lower than the cutoff frequency, $3 \mathrm{kHz}$, for the stop band of the system without local resonators. In the limit $M / m \rightarrow \infty$, the structure attenuates strongly even at frequencies much higher than the local resonance frequency, $\Omega$. However, the low frequency shift of the local resonance is limited in this case due to the overall mass constraint. Figure 7(b) shows the effect of changing the stiffness of the local resonators, while the masses are held constant. As seen in the figure, the frequency of the local resonance can be shifted lower when resonator stiffness decreases. However, the effectiveness of the local resonators progressively diminishes with the decrease in the stiffness, $K$.

In the analyses so far, all the local resonators in the structure had uniform mass and stiffness, thus the local resonance occurred at a single frequency. Here, we consider the effect of using non-uniform mass and stiffness for the resonators. In Fig. 8(a), we explore the transmittance of the system when the properties of the local resonators are not kept constant along the chain. This is achieved by sweeping the local natural frequency linearly from $500 \mathrm{~Hz}$ for the resonator attached to the first bead up to $1.92 \mathrm{kHz}$ for the resonator attached to the last bead, while keeping the mass of the resonator constant. In this case, we observe a much broader stop band, which is often desirable in practical vibration mitigation applications. Figure 8(a) also shows that, by reducing the
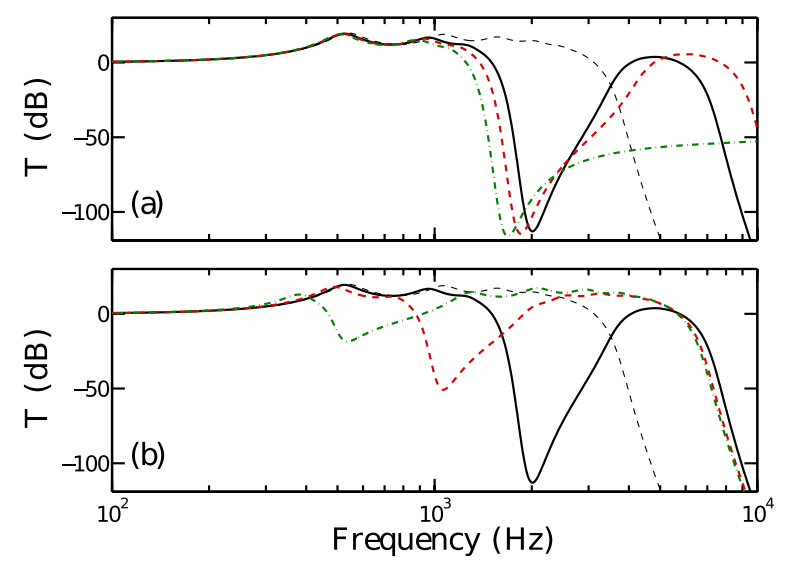

FIG. 7. Transmissibility of a finite structure consisting of ten beads with local resonators. In all diagrams, the black solid line refers to a structure with $m=45 \mathrm{~g}, M=101 \mathrm{~g}, k=20 \mathrm{~N} / \mu \mathrm{m}, \Omega / 2 \pi=1.92 \mathrm{kHz}$, and the gray dashed line to a structure without local resonators but with the mass of the beads equal to $m+M$. (a) The red dashed line refers to $m=25 \mathrm{~g}$ and the green dashed-dotted line to the limiting case $m \rightarrow 0, M=146 \mathrm{~g}$, while keeping the stiffness of the local resonators constant. (b) The red dashed line corresponds to $\Omega / 2 \pi=1000 \mathrm{~Hz}$ and the green dashed-dotted line to $\Omega / 2 \pi=500 \mathrm{~Hz}$, with $M=101 \mathrm{~g}$ in all cases. 

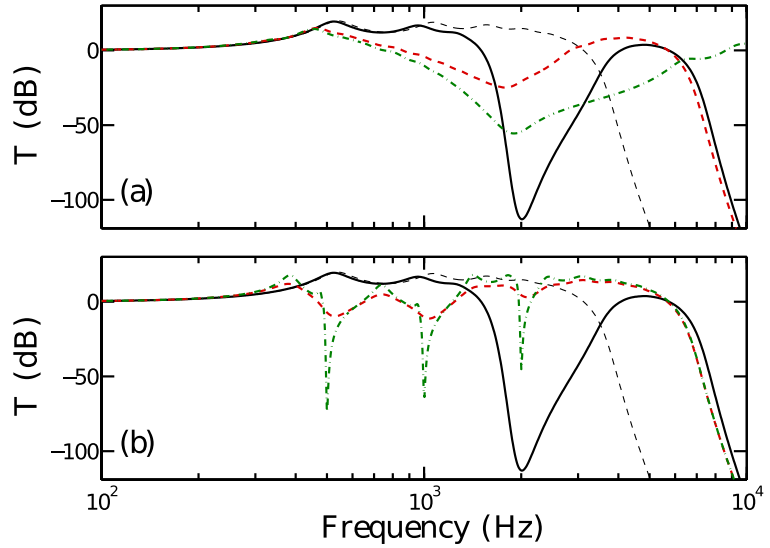

FIG. 8. Transmissibility of a finite structure consisting of ten beads with local resonators. In all diagrams, the black solid line refers to a structure with $m=45 \mathrm{~g}, M=101 \mathrm{~g}, k=20 \mathrm{~N} / \mu \mathrm{m}, \Omega / 2 \pi=1.92 \mathrm{kHz}$, and the gray dashed line to a structure without local resonators but with the mass of the beads equal to $m+M$. (a) A chain with variable local resonators with their eigenfrequencies changing linearly from $\Omega / 2 \pi=500 \mathrm{~Hz}$ to $1.92 \mathrm{kHz}$ with $M=101 \mathrm{~g}$ for all the local resonators (red dashed line) and with $M=141 \mathrm{~g}$, $m=5 \mathrm{~g}$ (green dashed-dotted line). (b) An heterogeneous chain with local resonators with eigenfrequencies located at $\Omega / 2 \pi=500 \mathrm{~Hz}, 1 \mathrm{kHz}$, and $2 \mathrm{kHz}$ with constant stiffness for all the local resonators and a loss factor of 0.2 (red dashed line) or 0.01 (green dashed-dotted line).
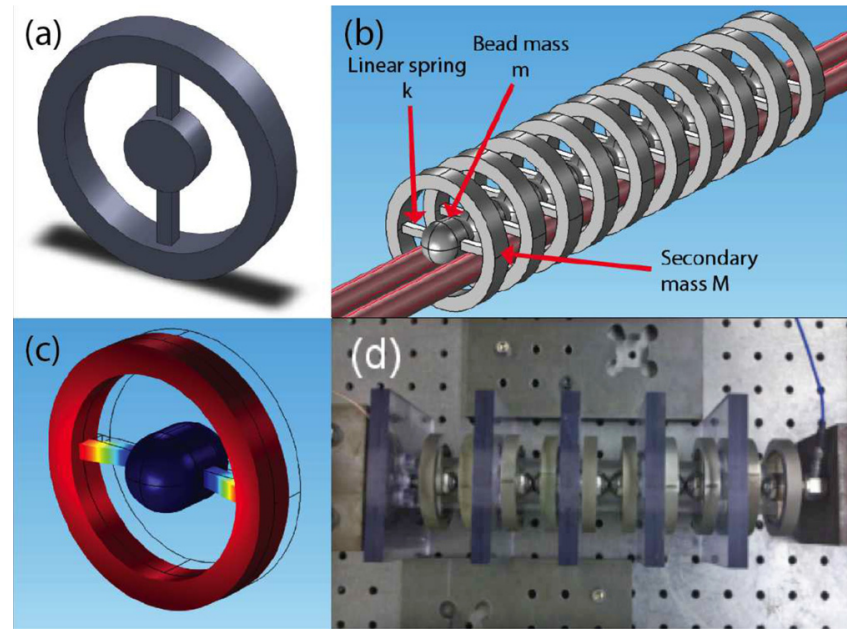

FIG. 9. (a) 3D model of the local ring resonator. ${ }^{22}$ (b) $3 \mathrm{D}$ representation of a chain of 10 beads with attached resonators. (c) Profile of the first eigenmode of the local resonator at $1.92 \mathrm{kHz}$. (d) Picture of the experimental setup. mass $m$ by $90 \%$ in favor of the mass of the local resonators, we can achieve deeper and broader attenuation for the whole frequency range from $500 \mathrm{~Hz}$ up to $6 \mathrm{kHz}$. Figure 8(b) shows another design, where attenuation of multiple tones can be achieved by distributing the resonator eigenfrequencies at 500,1000 , and $2000 \mathrm{~Hz}$.

\section{EXPERIMENTAL RESULTS}

In order to experimentally verify the vibration blocking ability of discrete mass-spring systems with local resonances, we have built an experimental setup consisting of a chain of spherical stainless steel beads, where each of the beads is attached to a ring shaped resonator also made of stainless steel. The ring resonator is shown in Fig. 9(a) and it has a fundamental mode of vibration at $1.92 \mathrm{kHz}$ with the profile shown in Fig. 9(c). The whole setup with the ten beads, the actuator on the left and the rigid base on the right are shown in Fig. 1(d), and schematically in Fig. 1(b). The force between the beads can be modeled as a Hertzian contact, which means that the force depends nonlinearly on the displacement. However, since the static overlap between the beads is much larger than the vibration amplitude, the equations of motion can be linearized and therefore the contact can be modeled as a linear spring of stiffness $k^{36,37}$

The attached local resonator has a primary eigenmode at $1.92 \mathrm{kHz}$. The profile of this mode, calculated with the commercial finite elements package COMSOL Multiphysics, is presented in Fig. 9(a). Since most of the motion of this eigenmode is parallel to the axis of the bead, we can model it as a linear spring-mass system attached to each bead, and vibrating in the same axis as the beads do. Therefore, the whole frequency response of the structure can be accurately described by Eq. (5).

Figure 10 presents the results of the transmittance for one and two beads system. The theoretical results have been scaled by a constant factor to match the driving amplitude of the experimental system. It can be seen that the shape and the position of the eigenmodes are well described by our theoretical model. A major discrepancy can be found at about $6 \mathrm{kHz}$, where an unexpected dip occurs. This dip is created by a higher order eigenmode of the local resonator, which can be modeled as an additional mass-spring system, resulting in an extra antiresonance at the natural frequency of the second spring-mass system. This result suggests a potential
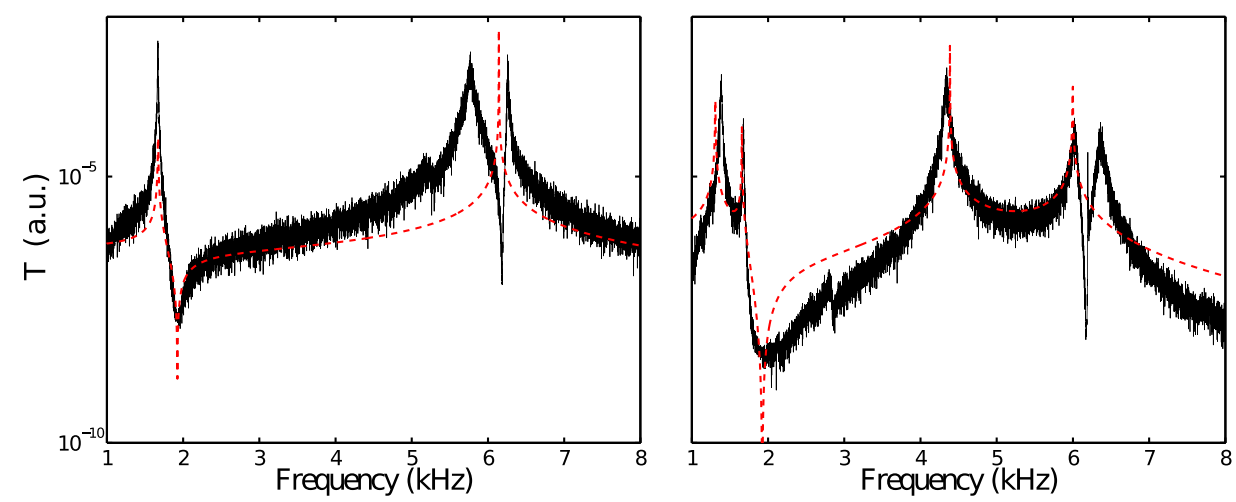

FIG. 10. Comparison between the experimental (black solid line) and the theoretical (red dashed line) results for a system consisting of one (left diagram) and two (right diagram) beads with $m=45 \mathrm{~g}$ and $k=25 \mathrm{~N} / \mu \mathrm{m}$ for the one bead, $k=16 \mathrm{~N} / \mu \mathrm{m}$ for the two beads system. The resonator parameters are $M=101 \mathrm{~g}, \Omega / 2 \pi=1.92 \mathrm{kHz}$. The theoretical line is scaled to match the excitation amplitude used in the experimental results. 


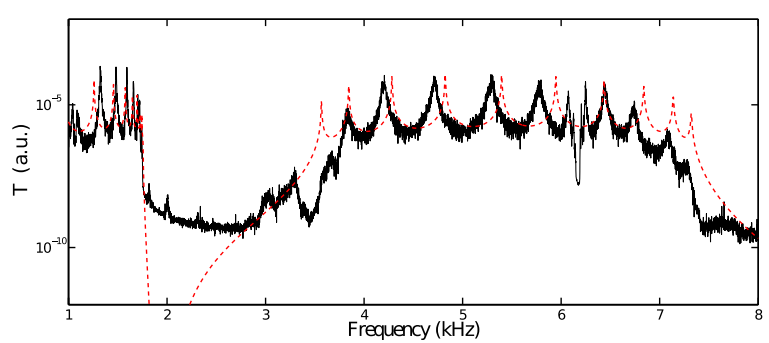

FIG. 11. Comparison between the experimental (black solid line) and the theoretical (red dashed line) results for a periodic arrangement of ten beads of $m=45 \mathrm{~g}, k=20 \mathrm{~N} / \mu \mathrm{m}$ and attached local resonators with $M=101 \mathrm{~g}$, $\Omega / 2 \pi=1.92 \mathrm{kHz}$.

vibration mitigation scheme based on using multiple eigenmodes of the same structure to block vibrations at various frequencies. However, detailed analysis of systems with multiple local resonances attached at each bead is beyond the scope of the present work.

The response of the full structure with the ten beads is shown in Fig. 11. We can see that there is a good qualitative agreement between the experimental results and the model. However, the position of the peaks is not captured correctly. The most probable explanation for this discrepancy is that asperites in the beads result in differences in the contact radii and therefore in different contact stiffnesses. However, the local resonator band gap edge at $1.76 \mathrm{kHz}$, as well as the stop band starting above $7.3 \mathrm{kHz}$ are well captured by the model.

\section{CONCLUSIONS}

We have studied the response of a one-dimensional metamaterial for vibration mitigation that exploits local resonances to filter low frequency excitations. The analyses included fundamental dynamic characteristics of local resonances along with an applied investigation of the efficacy of the metamaterial under mass and stiffness constraints. Finally, a practical implementation has been presented and built, and experimental results have been obtained to validate the analytical model. The ability to introduce a stopband at low frequency is desirable in practical vibration control problems. We have also shown that the shape of the stopband can be taylored to enhance the performance through strategic selection of non-uniform resonator parameters. Follow up studies will include systems containing multiple local resonances at each bead, as well as the effect of nonlinearity in the metamaterial. The interplay between periodicity, local resonances, and the intrinsic nonlinearity in these structures, originating from the Hertzian contact, provides a rich dynamic platform to be explored for vibration mitigation and energy trapping applications.

\section{ACKNOWLEDGMENTS}

We acknowledge support from this work from the United Technology Research Center and from the Army Research Office MURI Grant No. US ARO W911NF-09-1-0436.

${ }^{1}$ E. Shamonina and L. Solymar, Metamaterials 1, 12 (2007).

${ }^{2}$ R. W. Ziolkowski and N. Engheta, in Metamaterials (John Wiley \& Sons Inc., 2006), pp. 1-41.

${ }^{3}$ D. R. Smith, J. B. Pendry, and M. C. K. Wiltshire, Science 305, 788 (2004).

${ }^{4}$ N. Stefanou and A. Modinos, Phys. Rev. B 57, 12127 (1998).

${ }^{5}$ A. Yariv, Y. Xu, R. K. Lee, and A. Scherer, Opt. Lett. 24, 711-713 (1999).

${ }^{6}$ S. A. Maier, P. G. Kik, H. A. Atwater, S. Meltzer, E. Harel, B. E. Koel, and A. A. Requicha, Nature Mater. 2, 229 (2003).

${ }^{7}$ G. Gantzounis and N. Stefanou, Phys. Rev. B 74, 085102 (2006).

${ }^{8}$ Z. Liu, X. Zhang, Y. Mao, Y. Y. Zhu, Z. Yang, C. T. Chan, and P. Sheng, Science 289, 1734 (2000).

${ }^{9}$ S. H. Lee, C. M. Park, Y. M. Seo, Z. G. Wang, and C. K. Kim, Phys. Lett. A 373, 4464 (2009).

${ }^{10}$ A. Baz, New J. Phys. 11, 123010 (2009).

${ }^{11}$ H. H. Huang and C. T. Sun, New J. Phys. 11, 013003 (2009).

${ }^{12}$ S. Yao, X. Zhou, and G. Hu, New J. Phys. 12, 103025 (2010).

${ }^{13}$ Y. Cheng, J. Y. Xu, and X. J. Liu, Phys. Rev. B 77, 045134 (2008).

${ }^{14}$ N. Fang, D. Xi, J. Xu, M. Ambati, W. Srituravanich, C. Sun, and X. Zhang, Nature Mater. 5, 452 (2006).

${ }^{15}$ L. Hao, C. Ding, and X. Zhao, Appl. Phys. A 106, 807 (2012).

${ }^{16}$ J. Li and C. T. Chan, Phys. Rev. E 70, 055602 (2004).

${ }^{17}$ S. Guenneau, A. Movchan, G. Pétursson, and S. A. Ramakrishna, New J. Phys. 9, 399 (2007).

${ }^{18}$ F. Bongard, H. Lissek, and J. R. Mosig, Phys. Rev. B 82, 094306 (2010).

${ }^{19}$ X. Liu, G. Hu, C. Sun, and G. Huang, J. Sound Vib. 330, 2536 (2011).

${ }^{20}$ J. Li, L. Fok, X. Yin, G. Bartal, and X. Zhang, Nature Mater. 8, 931 (2009).

${ }^{21}$ G. W. Milton and J. R. Willis, Proc. R. Soc. London, Ser. A 463, 855 (2007).

${ }^{22}$ P. G. Kevrekidis, A. Vainchtein, M. Serra-Garcia, and C. Daraio, Phys. Rev. E 87, 042911 (2013).

${ }^{23}$ Z. Yang, H. M. Dai, N. H. Chan, G. C. Ma, and P. Sheng, Appl. Phys. Lett. 96, 041906 (2010).

${ }^{24}$ J. Jensen, J. Sound Vib. 266, 1053 (2003).

${ }^{25}$ G. Wang, J. Wen, and X. Wen, Phys. Rev. B 71, 104302 (2005).

${ }^{26}$ Y. Xiao, B. R. Mace, J. Wen, and X. Wen, Phys. Lett. A 375, 1485 (2011).

${ }^{27}$ Y. Xiao, J. Wen, and X. Wen, J. Phys. D: Appl. Phys. 45, 195401 (2012).

${ }^{28}$ Y. Xiao, J. Wen, and X. Wen, J. Sound Vib. 331, 5408 (2012).

${ }^{29}$ V. Romero-García, A. Krynkin, L. Garcia-Raffi, O. Umnova, and J. Sánchez-Pérez, J. Sound Vib. 332, 184 (2013).

${ }^{30}$ H. Frahm, "Device for damping vibrations of bodies," U.S. patent 989,958 (18 April 1911).

${ }^{31}$ K. Liu and J. Liu, J. Sound Vib. 284, 1181 (2005).

${ }^{32}$ C. Yilmaz and N. Kikuchi, J. Sound Vib. 291, 1004 (2006).

${ }^{33}$ S. J. Jang and Y. J. Choi, J. Sound Vib. 303, 343 (2007).

${ }^{34}$ F. Geniet and J. Leon, Phys. Rev. Lett. 89, 134102 (2002).

${ }^{35}$ M. I. Hussein and M. J. Frazier, J. Appl. Phys. 108, 093506 (2010).

${ }^{36}$ V. Nesterenko, Dynamics of Heterogeneous Materials (Springer, 2001).

${ }^{37}$ C. Daraio, V. F. Nesterenko, E. B. Herbold, and S. Jin, Phys. Rev. E 73, 026610 (2006). 